\title{
INC Neonatal Adverse Events Terminology
}

National Cancer Institute

\section{Source}

National Cancer Institute. INC Neonatal Adverse Events Terminology. NCI Thesaurus.

Code C154914.

A neonatal adverse event severity scale developed by the Clinical Pharmacology workgroup of INC to help researchers use a common approach when reporting adverse events (AEs). This work will enhance regulatory science for neonates by providing standardized terminology for adverse event reporting. 